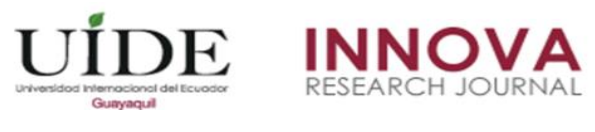

INNOVA Research Journal, ISSN 2477-9024

(Abril, 2018). Vol. 3, No.4 pp. 120-128

DOI: https://doi.org/10.33890/innova.v3.n4.2018.644

URL: http://revistas.uide.edu.ec/index.php/innova/index

Correo: innova@uide.edu.ec

\title{
Estudio de emisiones contaminantes producidas por un motor MEP con transmisión automática y transmisión manual
}

\section{Study of polluting emissions produced by an MEP engine with automatic transmission and manual transmission}

Julio César Leguísamo Milla

Santiago Fernando Celi Ortega

Universidad Internacional SEK del Ecuador, Ecuador

Marco Vinicio Noroña Merchán

Edwin Giovanny Puente Moromenacho

Universidad Internacional del Ecuador, Ecuador

Autor para correspondencia: julio.leguisamo@uisek.edu.ec; santiago.celi@uisek.edu.ec;

manoroname@uide.edu.ec; epuente@uide.edu.ec

Fecha de recepción: 21 de Octubre de 2017 - Fecha de aceptación: 15 de Abril de 2018

Resumen: Este estudio compara las emisiones contaminantes producidas en un motor MEP, el primero con transmisión manual y el segundo con transmisión automática. En un vehículo de la marca Chevrolet modelo SZ de cilindraje $2000 \mathrm{~cm}^{3}$ a 2800 metros de altura debido a que la altitud sobre el nivel del mar tiene un notable efecto en la formación de gases contaminantes porque esta variable modifica el ciclo termodinámico de operación y las condiciones de combustión Se realizó una prueba estacionaria y una prueba en ruta, en base a protocolos de pruebas establecidos por el Centro de Transferencia Tecnológica para la Capacitación e Investigación en Control de Emisiones Vehiculares (CCICEV). Los resultados obtenidos revelan que un vehículo con transmisión automática produce menor cantidad de emisiones contaminantes en la prueba estática y en la prueba en ruta que el mismo vehículo con transmisión mecánica. Existen gases contaminantes que se reducen en un alto porcentaje con respecto a la caja mecánica cuando se utiliza una automática.

Palabras Claves: emisiones; transmisión manual; transmisión automática; MEP; consumo combustible

\begin{abstract}
In this study we compare the contaminant emissions produced be an engine with triggered ignition, the first one with a manual transmission and the second one with an automatic transmission. For this study we have selected a Chevrolet vehicle model SZ with $2000 \mathrm{~cm}^{3}$ cylinders used in an altitude of 2800 meters over sea level, considering that this altitude has a notable effect in the formation of contaminant gases because this variable modifies the thermodynamic cycle of operation and the combustion conditions. Two different tests were conducted; a stationary test and a test in route considering the test protocols that are established by the Technological Transference Center for Training and Research of Vehicles Emissions (CCICEV). The results obtained by this investigation revealed that a vehicle with automatic transmission produces less quantity of contaminant emissions in the stationary test and in test in route than the same vehicle with manual transmission. There are polluting gases that are reduced in a high percentage with respect to the mechanical box when an automatic is used.
\end{abstract}


Key Words: emissions; manual transmission; automatic transmission; and engine with triggered ignition; combustion conditions

\section{Introducción}

Los elevados niveles de contaminación registrados en la actualidad han obligado a los diferentes organismos de control ambiental de los países desarrollados a establecer normas de emisiones de gases contaminantes en los vehículos cada vez más estrictas para poder comercializar sus vehículos. Razón por la cual se ha incrementado el equipamiento transmisiones automáticas para las emisiones contaminantes y mantener las prestaciones de torque y potencia solicitadas por los conductores. (Asociación de Empresas Automotrices del Ecuador AEADE, 2009). En las ciudades de altura como Quito que está a 2800 metros sobre el nivel del mar, se presenta una topografía muy irregular y se desconoce los valores de reducción de emisiones contaminantes.

La contaminación del aire constituye una de las amenazas más importantes para la salud pública. En el año 2012 la contaminación atmosférica causó la muerte prematura de aproximadamente 3.7 millones de personas a nivel mundial (Vega, 2015). A nivel mundial de acuerdo con las últimas estimaciones realizadas por el grupo Intergubernamental de Expertos sobre el cambio Climático (IPCC), la emisión asociada a la quema de combustibles fósiles alcanza un valor del 56,6\% del total, lo que equivale a más de 27 GTon $\mathrm{C}_{2}$-eq (IPCC, 2007)

Además, se debe recalcar que la demanda de combustible sigue en aumento debido a que otros tipos de energía alternativas no se han consolidado en el mercado, teniendo como consecuencia en el futuro escases de combustible. Considerando que el parque automotor tiene un incremento anual del $1,1 \%$ en el consume de combustible y mediante proyecciones se dice que será el mayor consumidor con el 63\% en las próximas tres décadas. (Iodice, 2016)

En la actualidad se han realizado estudios de emisiones y consumo de combustible en vehículos con transmisión automática como se menciona en (Iñiguez, 2017) donde se compara las emisiones producidas con un vehículo Ford F150 con transmisión automática utilizando gasolina común y un combustible alternativo E5 mediante pruebas estáticas y dinámicas. De donde se obtuvo como resultado la reducción de emisiones contaminantes de $\mathrm{CO}, \mathrm{HC} \mathrm{y} \mathrm{CO}_{2}$, pero no se han relacionado en el mismo auto con transmisión manual.

En su estudio Grégoire S. (2013) desarrolla una investigación en la cual determina el consumo de combustible y las emisiones contaminantes a nivel del mar en un vehículo Toyota Camry 2007 con transmisión automática en rutas urbanas y determinan con una conducción ecológica se reduce el consumo de combustible entre un $7 \%$ y las emisiones se reducen el dióxido de carbono $\left(\mathrm{CO}_{2}\right)$ en $2 \%$, el monóxido de carbono $(\mathrm{CO})$ en $3 \%$.

En el artículo Shushan, B. (2007) indica como el control de la transmisión automática con la ayuda pedal del acelerador electrónico coordina la apertura de la aleta de aceleración para satisfacer la petición de poder del conductor manteniendo un cambio de marcha optima y maximizando el ahorro de combustible mediante pruebas de torque y potencia en el laboratorio demostrando que la caja automática es más eficiente y amigable con el ambiente que una caja manual. 
En el estudio (Tipanluisa, 2017) se realiza una investigación entre la calidad del combustible y las emisiones contaminantes producidas a 0 y 2500 metros con respecto al nivel del mar y se obtienen datos que garantizan la repetitividad y confiabilidad debido al método utilizado que ha sido desarrollado por el Centro de investigación y Control de Emisiones Vehiculares de la Escuela Politécnica Nacional y se obtuvieron los efectos significativos que se interrelacionan entre los factores que inciden en las variables de respuesta de las emisiones contaminantes.

El propósito de este estudio es determinar el efecto de la altitud a 2800 metros con respecto al nivel del mar en las emisiones producidas en un vehículo con transmisión manual en relación con un vehículo con transmisión automática, el cual se obtiene realizando una prueba estacionaria y una prueba en ruta, que nos permiten determinar las emisiones contaminantes de $\mathrm{CO}$, $\mathrm{CO}, \mathrm{HC}$ y $\mathrm{NOX}$, para fomentar en la población la compra de vehículos equipados con transmisiones automáticas para reducir las emisiones contaminantes.

\section{Materiales}

Los vehículos utilizados en las pruebas son un Chevrolet SZ con transmisión manual y otro con transmisión automática, que no han sido modificados, las especificaciones se pueden encontrar en la tabla 1.

Tabla 1 Especificaciones técnicas de los vehículos.

\begin{tabular}{lll}
\hline Datos & Vehículo 1 & Vehículo 2 \\
\hline Combustible & Gasolina Super & Gasolina Super \\
Cilindraje motor & $1995 \mathrm{cc}$ & $1995 \mathrm{cc}$ \\
Transmisión & Automática & Manual \\
Torque & $183 / 4000 \mathrm{Nm} / \mathrm{rpm}$ & $183 / 4000 \mathrm{Nm} / \mathrm{rpm}$ \\
Potencia & $140 / 6000 \mathrm{CV} / \mathrm{rpm}$ & $140 / 6000 \mathrm{CV} / \mathrm{rpm}$ \\
Cilindros & 4 en línea & 4 en línea \\
\hline
\end{tabular}

FUENTE: Manual de usuario SZ 2.0

Se usó un analizador de gases estático marca MAHA, analizador de gases portable, marca NEXTECH, GPS marca GARMIN, escáner marca CARMAN, medidores de flujo fabricación CCICEV y convertidor de corriente marca Schumacher.

\section{Métodos}

Se realizaron cinco pruebas en cada condición del experimento y en las cuales se ejecutó todos los ciclos establecidos y se obtuvieron los datos necesarios a para determinar las emisiones contaminantes.

Para determinar si los datos obtenidos en la experimentación son fiables debido a que pueden presentar desviaciones, con respecto al objetivo específico y se pueden manifestar en 
forma de datos inexactos y se puede producir una excesiva variabilidad respecto a los valores deseables, se realizó el control de rangos y se determinó que las mediciones obtenidas son fiables.

Las pruebas se realizaron a una altura referencial de 2800 metros sobre el nivel del mar y temperatura ambiente de $15^{\circ} \mathrm{C}$, en los dos vehículos y se establece si la caja automática con relación a la caja manual ofrece una ventaja adicional con respecto a las variables seleccionadas.

\section{Análisis de resultados}

\section{Emisiones del motor en la prueba estática}

En las siguientes tablas se puede observar los valores de emisiones obtenidas en la prueba estática y en ruta de los dos vehículos el equipado con transmisión manual y con transmisión automática.

Tabla 2 Valores de emisiones en prueba estática a ralenti $750 \mathrm{rpm}$

\begin{tabular}{lllll}
\hline Transmisión & $\mathbf{C O}(\boldsymbol{\%})$ & $\mathbf{C O}_{2}(\boldsymbol{\%})$ & $\mathbf{H C}(\mathbf{p p m})$ & $\mathbf{N O x}(\mathbf{p p m})$ \\
\hline Manual & 0.28 & 14.70 & 116.50 & 5.00 \\
Automática & 0.04 & 14.70 & 51.00 & 0.00 \\
\hline
\end{tabular}

Tabla 3 Valores de emisiones en prueba estática a $2500 \mathrm{rpm}$

\begin{tabular}{lllll}
\hline Transmisión & CO (\%) & CO2 $_{2}(\%)$ & HC(ppm) & NOx(ppm) \\
\hline Manual & 0.38 & 14.55 & 82.50 & 46.00 \\
Automática & 0.01 & 14.55 & 34.50 & 1.50 \\
\hline
\end{tabular}

Tabla 3. Valores de volumen de emisiones en prueba dinámica vehículo transmisión automática

\begin{tabular}{|c|c|c|c|c|c|}
\hline Dato & Tiempo & $\mathrm{CO}$ & $\mathrm{HC}$ & $\mathrm{CO2}$ & Nox \\
\hline No. & (s) & $(\%)$ & (ppm) & $(\%)$ & (ppm) \\
\hline 5 & 50 & 0,23 & 24 & 13,6 & 355 \\
\hline 10 & 100 & 3,4 & 48 & 9,6 & 429 \\
\hline 15 & 150 & 0,39 & 33 & 10,9 & 404 \\
\hline 20 & 200 & 0,24 & 46 & 9,4 & 479 \\
\hline 25 & 250 & 0,43 & 100 & 11,2 & 417 \\
\hline 30 & 300 & 0,33 & 51 & 12,9 & 337 \\
\hline 31 & 310 & 4,08 & 92 & 13,1 & 385 \\
\hline
\end{tabular}

Tabla 4. Valores de volumen de emisiones en prueba dinámica vehículo transmisión mecánica

\begin{tabular}{rrrrrr}
\hline Dato No. & Tiempo (S) & CO (\%) & HC (ppm) & CO2 (\%) & Nox (ppm) \\
\hline $\mathbf{1}$ & 10 & 0,45 & 278 & 13,4 & 71 \\
$\mathbf{5}$ & 50 & 2,56 & 102 & 13,1 & 409 \\
$\mathbf{1 0}$ & 100 & 5,37 & 136 & 10,4 & 484 \\
$\mathbf{1 5}$ & 150 & 0,07 & 40 & 1 & 67 \\
\hline
\end{tabular}


En el análisis de los resultados de la prueba estática y en ruta, se realizó el respectivo control de rangos para garantizar la fiabilidad y confiabilidad de los datos obtenidos con respecto a las emisiones de gases de los dos vehículos de prueba.

\section{Emisiones de CO}

Realizado el control de rangos y el promedio de la tabla 6 se puede observar que las emisiones de $\mathrm{CO}$ en la prueba estática a $750 \mathrm{rpm}$, producidas por el vehículo equipado con transmisión automática son un 0,24\% menores comparadas con las emisiones del vehículo con transmisión manual. Además, a $2500 \mathrm{rpm}$ las emisiones del vehículo con transmisión manual aumentan a 0,30\% y del vehículo con transmisión automática se reduce a 0,01\%.

\section{Emisiones de $\mathrm{CO}_{2}$}

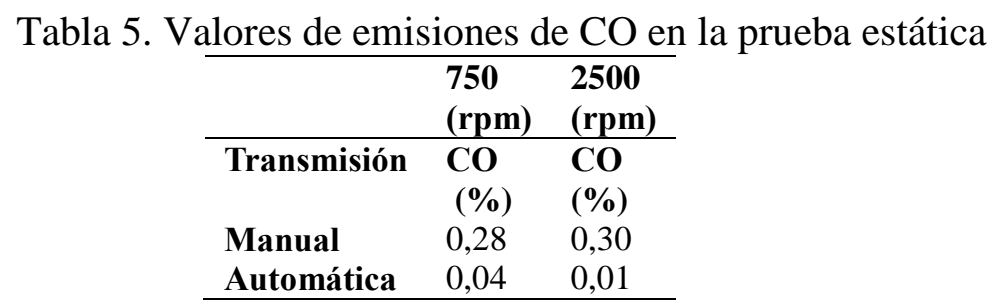

En la tabla 7 se puede observar que el promedio de contaminación del $\mathrm{CO}_{2}$ de los dos vehículos es el mismo.

Tabla 6. Valores de emisiones de $\mathrm{CO}_{2}$ en la prueba estática

\begin{tabular}{lll}
\hline & $\begin{array}{l}\mathbf{7 5 0} \\
(\mathbf{r p m})\end{array}$ & $\begin{array}{l}\mathbf{2 5 0 0} \\
(\mathbf{r p m})\end{array}$ \\
\hline Transmisión & $\mathrm{CO}_{\mathbf{2}}$ & $\begin{array}{l}\mathrm{CO}_{2} \\
(\mathbf{\%})\end{array}$ \\
Manual & 14,70 & 14,55 \\
Automática & 14,70 & 14,55 \\
\hline
\end{tabular}

\section{Emisiones de HC}

Con respecto a las emisiones de $\mathrm{HC}$ que genera el vehículo con transmisión manual se puede observar una diferencia muy grande de 65,50 ppm (partículas por millón) a 750 rpm y los 48 ppm (partículas por millón) a $2500 \mathrm{rpm}$, con lo que se puede observar una reducción de las emisiones de $\mathrm{HC}$, el vehículo con transmisión automática emite el 43,77\% de las emisiones totales del vehículo con transmisión manual a ralentí. A $2500 \mathrm{rpm}$ las emisiones de $\mathrm{HC}$ del vehículo con transmisión automática corresponden aproximadamente a la mitad de las emisiones producidas por el auto con transmisión manual.

Tabla 7. Valores de emisión de HC en prueba estática

\begin{tabular}{lll}
\hline & $\begin{array}{l}\mathbf{7 5 0} \\
(\mathbf{r p m})\end{array}$ & $\begin{array}{l}\mathbf{2 5 0 0} \\
(\mathbf{r p m})\end{array}$ \\
\hline Transmisión & $\mathbf{H C}$ & $\mathbf{H C}$ \\
& $(\mathbf{p p m})$ & $(\mathbf{p p m})$ \\
Manual & 116,50 & 82,50 \\
Automática & 51,00 & 34,50 \\
\hline
\end{tabular}




\section{Emisiones de NOx}

Los valores obtenidos de las emisiones de óxidos de nitrógeno (NOx), no representan el valor real de este contaminante, ya que estos deben ser evaluados en una prueba bajo carga a pesar de esta condición se puede observar una gran diferencia a $2500 \mathrm{rpm}$ donde la emisión de NOx del vehículo con transmisión mecánica es 30 veces mayor.

\begin{tabular}{cll} 
Tabla 8. Valores de emisión de & NOx en prueba estática \\
\cline { 2 - 3 } & $\begin{array}{l}\mathbf{7 5 0} \\
(\mathbf{r p m})\end{array}$ & $\begin{array}{l}\mathbf{2 5 0 0} \\
\mathbf{( r p m )}\end{array}$ \\
\hline Transmisión & $\begin{array}{c}\text { NOx } \\
\text { (ppm) }\end{array}$ & $\begin{array}{l}\text { NOx } \\
\text { (ppm) }\end{array}$ \\
& 5,00 & 46,00 \\
Manual & 0,00 & 1,50 \\
\hline
\end{tabular}

\section{Análisis de prueba en ruta}

\section{Emisiones de CO}

Como se puede observar en la figura 1 el vehículo con transmisión mecánica produce mayor emisión de $\mathrm{CO}$ y se puede observar valores más irregulares con un valor máximo de que emisión de $6,04 \%$, valor mínimo de $0,22 \%$ y una media de $1,185 \%$; en cambio se puede observar en el vehículo con transmisión automática un valor máximo de $1,94 \%$, un mínimo de $0 \%$ y una media $0,106 \%$. Lo que demuestra que el vehículo con transmisión automática produce menos emisiones contaminantes.

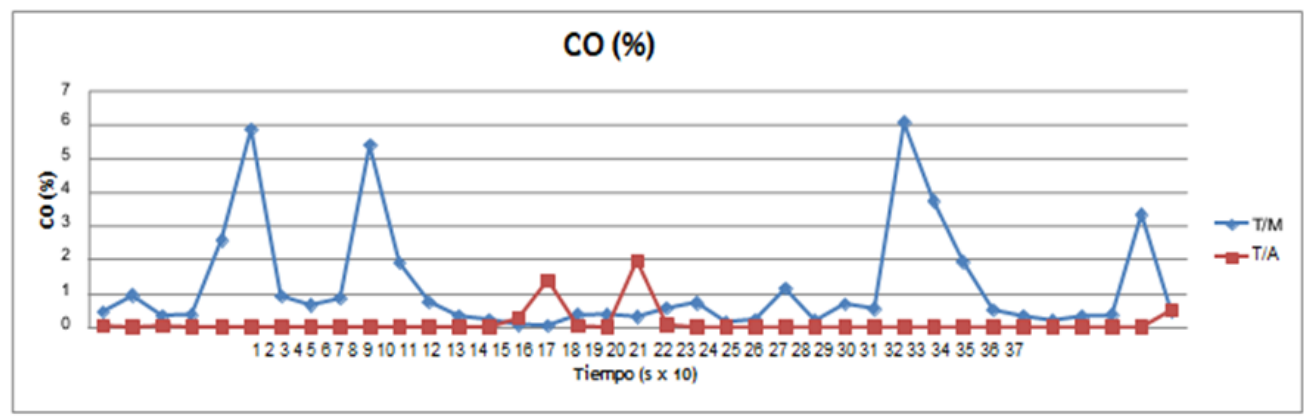

Figura 1. Comparación emisiones de $\mathrm{CO}$ en vehículos con transmisión automática y manual

\section{Emisiones de $\mathrm{CO} 2$}

La figura 2 muestra que las emisiones de $\mathrm{CO}_{2}$ son similares pero el mayor valor de $\mathrm{CO}_{2}$ corresponde al vehículo con transmisión manual con valor de 14,1\% mientras que en el vehículo con transmisión automática el mayor valor corresponde 13,1\%, los valores mínimos corresponden a $0,4 \%$ el manual y $4,8 \%$ el automático, y como promedio el automático indica $10,98 \%$ y el manual 10,654\%. Podemos observar que durante todo el ciclo el vehículo con transmisión manual emite menor cantidad de $\mathrm{CO}_{2}$. 


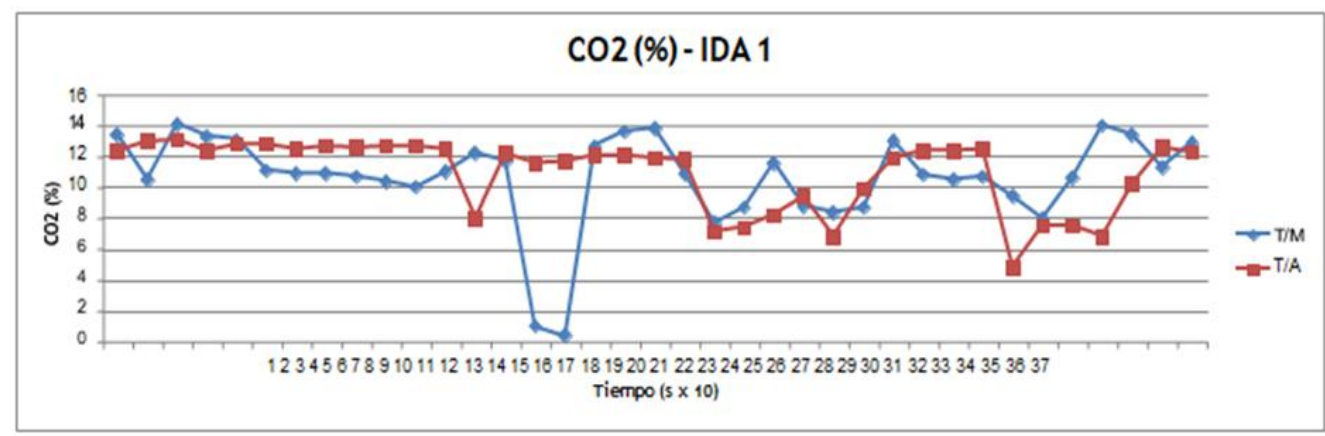

Figura 2. Comparación emisiones de $\mathrm{CO}_{2}$ en vehículos con transmisión automática y manual

\section{Emisiones de $\mathrm{HC}$}

En las emisiones de HC el valor más elevado es de 278 ppm que corresponde al vehículo con caja manual y este mismo auto su valor más bajo es 40 ppm, mientras que en el vehículo con transmisión automática el valor más alto es de $72 \mathrm{ppm}$, el mínimo de $29 \mathrm{ppm}$. El promedio es de 42,628 ppm en el auto con transmisión manual y 372,946 en el auto con transmisión manual. Además, se puede observar que las emisiones de $\mathrm{HC}$ se mantienen más estables y con valores más bajos en el auto con caja automática como se indica en la figura

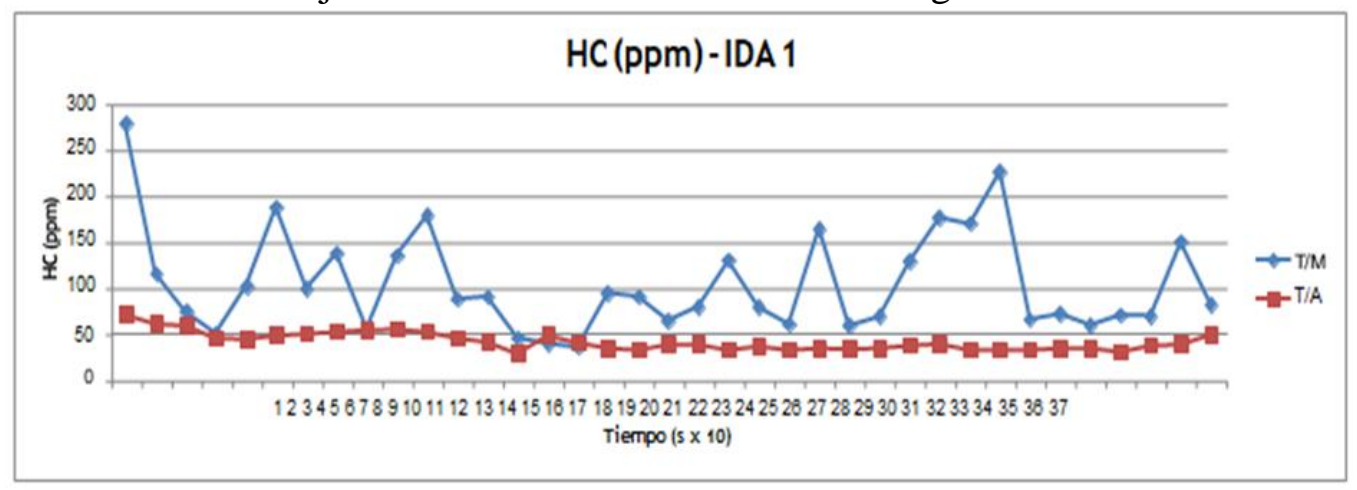

Figura 3. Comparación emisiones de HC en vehículos con transmisión automática y manual

\section{Emisiones de NOx}

Para el caso de los óxidos de nitrógeno se puede apreciar que el mayor valor lo produce la transmisión automática con 1119 comparando con el mayor valor de 704 de la caja manual, mientras que el menor valor de la caja automática es $0,0 \quad$ y el de la caja manual es 29. Analizando el promedio se puede determinar que el de la caja automática es 155,907 y de la caja manual 372,946 que es menor y en todo el ciclo la caja automática produce menos NOx. 


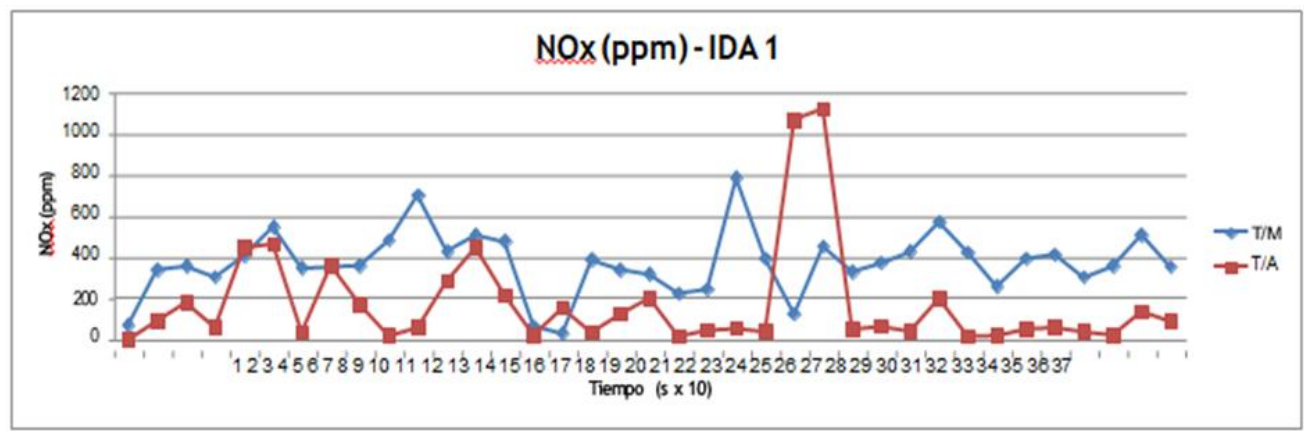

Figura 4. Comparación emisiones de NOx en vehículos con transmisión automática y manual

\section{Conclusiones}

En el presente estudio se demuestra una clara reducción de emisiones contaminantes producidas por el motor de combustión interna al utilizar una transmisión automática a 2800 metros de altura con respecto al mismo vehículo equipado con una transmisión manual.

En las pruebas estáticas tenemos: en CO una reducción del 75\% en ralentí y una reducción del $96 \%$ a $2500 \mathrm{rpm}$ con respecto a las emisiones producidas con un vehículo con transmisión manual; el $\mathrm{CO}_{2}$ indica los mismos valores en los dos casos. Con respecto a los $\mathrm{HC}$ hay una reducción del $56,23 \%$ en ralentí y a 2500 rpm hay una reducción del 58,18\% con respecto a las emisiones producidas por el vehículo con la transmisión mecánica. Las emisiones de NOx del vehículo con transmisión automática son menores que el vehículo con transmisión manual, pero estas no se las consideran debido a que el vehículo no está sometido a carga.

Las pruebas en ruta indican que las emisiones de $\mathrm{CO}$ son menores en un $91,50 \%$ en el vehículo con transmisión automática con respecto a la caja manual, en los $\mathrm{CO}_{2}$ los valores de emisión son muy similares con un valor de $10,98 \%$ para el auto con transmisión automática y $10,658 \%$ con transmisión manual pero el ciclo de emisión es más estable para el automático. En los HC existe una reducción del 88,56\% con respecto a las emisiones producidas por la transmisión mecánica. Los NOx se reducen en un 58,19\% con respecto a la caja mecánica cuando se utiliza una automática.

\section{Bibliografía}

El Petróleo, El recorrido de la energía. (2002). Madrid INNOVA, 1-19.

Grégoire, S. (2013). Fuel consumption and gas emissions of an automatic transmission vehicle following simple eco-drinving instructions on urban roads. Centre for accident research and road safety - Queensland University of Technology, Kelvin Grove, Queensland, Australia.

Grupo de Síntesis Orgánica. (2011). Alcanos Estructura y Nomenclatura. Sinorg, 1-34.

MAZDA. (2013). Workshop Manual Engine CX-5. U.S.A. 
Iñiguez, J. (2017). Study of Polluting Emissions Produced by an Otto Engine with the Use of Gasoline and Fuel Based on 95\% Gasoline and 5\% Ethanol. Innova Research Journal 2017, Vol 2, No. 12,11-18. Universidad Internacional. Quito Ecuador

Iodice, P. (2016). Effect of etanol. gasoline blends on CO and HC emissions in last generation SI within the cold-start transient: An experimental investigation. Aplied Energy, 182

IPCC. (2007). Intergovernamental Panel on Climate Change.

Lapuerta, M., O. Armas y J. R. Agudelo. (2005). Estudio del efecto de la altitud sobre las prestaciones de motores de combustión interna, IV Jornadas Nacionales de Ingeniería Termodinámica, La Rioja, España

Robles, L., \& Martínez, J. (Diciembre de 2010). Estudio del comportamiento de las variables de un motor de inyección electrónica respecto a la altura sobre el nivel del mar. Escuela Politecnica Nacional. Quito, Pichincha, Ecuador.

Shushan, B. (2007). Control of Integrated Powertrain With Electronic Throttle and Automatic Transmission. IEEE Transactions on Control systems technology

Soares, S. M. C. y J. R. Sodré. (2002). Effects of atmospheric temperature and pressure on the performance of a vehicle. Proc. Instn. Mech. Engrs., $216($ D) D08201.

Suarez, M. (2012). Interaprendizaje de Probabilidades y Estadística Inferencial con Excel Winstast y Graph. Ibarra, Imbabura, Ecuador: M \& V.

Tecnum. (2002). Ciencias de la tierra, Sustancias que contaminan la atmósfera. Gipuzkoa: Tecnun.

Tipanluisa, L. (2017). Emisiones Contaminantes de un Motor de Gasolina Funcionando a dos Cotas de Combustibles de dos Calidades. Información Tecnológica Vol. 28(1), 3-12

Vega, D. (2015). Inventario de emisiones atmosféricas del tráfico vehicular en el Distrito Metropolitano. Avances Ciencia e Ingeniería 2. 